\title{
Murine double minute 2 predicts response of advanced esophageal squamous cell carcinoma to definitive chemoradiotherapy
}

Hiroshi Okamoto ${ }^{1 *}$, Fumiyoshi Fujishima², Takashi Kamei ${ }^{1}$, Yasuhiro Nakamura ${ }^{3}$, Yohei Ozawa ${ }^{1,3}$, Go Miyata ${ }^{1}$ Toru Nakano ${ }^{1}$, Kazunori Katsura ${ }^{1}$, Shigeo Abe ${ }^{1}$, Yusuke Taniyama ${ }^{1}$, Tadashi Sakurai ${ }^{1}$, Jin Teshima ${ }^{1}$, Makoto Hikage $^{1}$, Hironobu Sasano ${ }^{2,3}$ and Noriaki Ohuchi ${ }^{1}$

\begin{abstract}
Background: Definitive chemoradiotherapy (dCRT) has recently become one of the most effective therapies for the treatment of esophageal squamous cell carcinoma (ESCC). However, it is also true this treatment has not been effective in all patients. Therefore, it is very important to evaluate the surrogate marker of dCRT in order to improve clinical outcomes of patients with ESCC. On the other hand, our previous study had suggested that murine double minute 2 (MDM2) and p16 were associated with chemoradioresistance in ESCC.

Methods: We selected pretreatment biopsy specimens of ESCC patients from our prospective clinical study on dCRT. Seventy-nine cases histologically diagnosed as ESCC were used. We immunohistochemically investigated these specimens using antibodies against MDM2, p53, p16, and Ki-67.

Results: The patients included 68 males and 11 females with a mean age of 63.3 years. The number of patients in each clinical stage was as follows: 22 in c-Stage I; 17 in c-Stage II; and 40 in c-Stage III. cT, cN, and cStage were significantly more advanced in the Failure group (including patients with persistent and recurrent disease after $\mathrm{dCRT}$ ) than in the complete response (CR) group (patients with persistent CR after dCRT). The clinical stage inversely correlated with the $C R$ rate and the rescue rate after failure. The overall survival rate was significantly worse in the patients with advanced $\mathrm{CT}, \mathrm{CN}$, and $\mathrm{cStage}$ levels, and in the Failure group. MDM2 positivity was significantly higher in the Failure group than in the CR group in CStagellI $(P=0.014)$. The number of patients with an absence of p16 immunoreactivity was significantly higher in the Failure group than in the CR group in CStagelll $(P=0.010)$ but not in cStagel or cStagell. Moreover, the overall survival with a Ki-67 $\geq 33.7 \%$ was significantly better than that with $<33.7 \%$ for patients in cStagelll $(P=0.024)$.

Conclusions: The results of this study suggested that MDM2 and p16 are predictive markers for chemoradioresistance in CStagelll ESCC and Ki-67 is a prognostic marker following dCRT in CStagelll ESCC. These issues could contribute to the formulation of treatment strategy for patients with advanced ESCC.
\end{abstract}

Keywords: Esophagus, Squamous cell carcinoma, MDM2, p53, p16, Ki-67, Chemoradiotherapy, Chemoradioresistance, Chemoradiosensitivity

\footnotetext{
*Correspondence: hi-ok-0531@med.tohoku.ac.jp

'Department of Advanced Surgical Science and Technology, Graduate School of Medicine, Tohoku University, 1-1 Seiryo-machi, Aoba-ku, Sendai 980-8574, Japan

Full list of author information is available at the end of the article
} 


\section{Background}

Definitive chemoradiotherapy (dCRT) has recently become one of the most effective therapies for esophageal squamous cell carcinoma (ESCC) [1]. The clinical outcomes with this treatment have been comparable with surgery alone [2]. We also reported that dCRT for patients with ESCC was comparable or even superior to surgery alone in terms of survival and quality of life [3]. However, treatment failures have also occurred following dCRT. Salvage surgery for these cases could be effective for selected patients, but this treatment has high morbidity and mortality [4]. Therefore, it has become important to estimate the possible response of ESCC to dCRT before treatment. On the other hand, by investigating the surgical specimens of salvage esophagectomies after dCRT, our previous study had suggested that murine double minute 2 (MDM2) and p16 are associated with chemoradioresistance in ESCC [5]. MDM2 directly interferes with the transcriptional activity of p53 and promotes p53 degradation by the addition of ubiquitin [6,7]. Overexpression of MDM2 has also been reported to be associated with development of radioresistance in several tumors [8]. p16 is a cyclin-dependent kinase inhibitor and its inactivation is related to carcinogenesis $[9,10]$. The purpose of this study was to explore whether MDM2 and p16 expression in the pretreatment biopsy specimens of ESCC patients could predict the response to dCRT or the survival of the patients after dCRT. We investigated this issue using immunohistochemical staining for MDM2, p53, p16, and Ki-67.

\section{Methods}

\section{Patients and tissue samples}

We selected the pretreatment biopsy specimens of ESCC patients from our prospective clinical study on CRT [3]. Briefly, eligible patients in the study were aged 20-80 years with previously untreated, T1-3 N0-3 M0 (the 7th edition of the Union for International Cancer Control system [11]), and histologically confirmed ESCC of the thoracic esophagus. The pretreatment evaluations included a barium meal, an esophagogastroduodenoscopy, a neck, chest, and abdominal computed tomography (CT), and a 2-[fluorine-18] fluoro-2-deoxy-D-glucose positron emission tomography (FDG-PET) when needed. Finally, we selected the pretreatment biopsy specimens of 79 patients, who had been histologically diagnosed with ESCC, according to the criteria among 119 cases examined.

\section{Definitive chemoradiotherapy and salvage esophagectomy}

The CRT protocol of the prospective study basically followed the protocol of the Japan Clinical Oncology Group trial 9906 [1]. This protocol consisted of the following components: (1) 2 cycles of an intravenous cisplatin infusion $\left(40 \mathrm{mg} / \mathrm{m}^{2}\right)$ on days 1 and 8 , (2) a continuous intravenous infusion of 5-fluorouracil (400 mg/ $\mathrm{m}^{2}$ ) over 24 hours on days $1-5$ and 8-12 every 5 weeks, and (3) concurrent radiotherapy (60 Gy in 30 fractions over a period of 8 weeks including a 2 -week remission following the administration of $30 \mathrm{~Gy}$ ). Salvage esophagectomy for patients with persistent or recurrent disease was performed to improve outcomes as soon as persistent or recurrent disease was confirmed, if we could obtain the informed consent from the patients and the patients were fit for the surgery [3].

\section{Immunohistochemistry}

Immunohistochemical staining was performed using the streptavidin-biotin complex method. In brief, serial 4- $\mu \mathrm{m}$-thick sections were deparaffinized and immersed in $3.0 \%$ hydrogen peroxide in methanol for $10 \mathrm{~min}$ at room temperature (RT) to block the endogenous peroxidase activity. For antigen retrieval, the slides for MDM2, p16, and Ki-67 were heated using an autoclave at $121^{\circ} \mathrm{C}$ for $5 \mathrm{~min}$ in $0.01 \mathrm{M}$ citrate buffer ( $\mathrm{pH} \mathrm{6.0).} \mathrm{The}$ slides for $\mathrm{p} 53$ were heated in a microwave at $95^{\circ} \mathrm{C}$ for $15 \mathrm{~min}$ in $0.01 \mathrm{M}$ citrate buffer ( $\mathrm{pH}$ 6.0). Then, the slides were incubated in 1\% normal rabbit serum for $30 \mathrm{~min}$ at RT to decrease the nonspecific antibody binding. Subsequently, the slides were incubated at $4^{\circ} \mathrm{C}$ overnight with mouse monoclonal antibody against MDM2 (SMP14; Santa Cruz Biotechnology Inc., CA, USA; diluted 1/1000), p53 (DO-7; Nichirei Biosciences Inc.; diluted 1/100), p16 (G175-1239; BD Biosciences; diluted 1/100), and Ki-67 (MIB-1; Dako; diluted 1/300). The next day, the sections were incubated separately with biotinylated antimouse immunoglobulin (Nichirei Biosciences Inc.) as a secondary antibody and with peroxidase-labeled streptavidin (Nichirei Biosciences Inc.) for $30 \mathrm{~min}$ at RT. The antigen-antibody complexes were visualized with 3,3'diaminobenzidine.

The percentage of MDM2-, p53-, and Ki-67-positive nuclei, and p16-positive nuclei and/or cytoplasm of tumor cells was evaluated by $\times 400$ magnification microscopy. When determining the cut-off values, we identified the values for abnormal expression as follows: p53 $\geq 10 \%$ [12] and $\mathrm{p} 16<5 \%$ [13]. The evaluation was performed by two of the authors ( $\mathrm{HO}$ and $\mathrm{FF}$ ) who were blinded to the relevant clinical information of the patients examined in this study.

\section{Statistical analyses}

All statistical analyses were performed using JMP Pro Version 9.0.2 (SAS Institute Inc., Cary, NC, USA). Continuous data were analyzed using Student's $t$-test or the Mann-Whitney $U$-test. Categorical data were evaluated using Pearson's chi-square test, Fisher's exact test, or the Mann-Whitney $U$-test as appropriate. Normality was assessed using the Shapiro-Wilk test. Equality of variances was evaluated using the F test. Overall survival 
(OS) curves were determined by the Kaplan-Meier method, and a log-rank test was used to compare the survival curves. A $P$ value of $<0.05$ was considered statistically significant. The patient survival time was determined from the date of the start of treatment until death or the last followup examination.

This study was approved by the Ethical Committee of Tohoku University Hospital (accession number 2011-596).

\section{Results}

\section{Patient characteristics and clinical courses}

Table 1 summarizes the clinicopathological findings of the patients examined in this study. The patients included 68 males and 11 females with a mean age of 63.3 years (range, 43-79 years). The number of patients in each clinical stage was as follows: 22 in c-Stage I; 17 in c-Stage II (7, T3N0; 6, T1N1; 4, T2N1); and 40 in c-Stage III (35, T3N1; 5, T3N2). The CR (complete response) group contained the patients who were determined to have a CR after dCRT and in whom CR had been continued. Failure group included the patients with persistent and recurrent disease after dCRT. The clinical courses of the patients according to clinical stage are shown in Figure 1. The clinical stage inversely correlated with the $\mathrm{CR}$ rate and the rescue rate following the therapeutic failure. Table 1 also shows that the patients with more advanced $\mathrm{cT}, \mathrm{cN}$, and cStage levels had more possibility to lead to Failure group rather than CR group. Moreover, such patients had a significantly worse prognosis compared with CR group (Table 2).

\section{Comparison of marker expression between the CR group and failure group}

The MDM2-positive rate in the Failure group was significantly higher than that in CR group in cStageIII $(P=$ $0.014)$. This was not the case in the entire group, or in those patients with cStageI or cStageII disease (Figure 2A). The p53 expression did not demonstrate any correlations between the CR group and the Failure group (Table 3). The number of patients that were p16 negative was significantly higher in the Failure group than in the $C R$ group in the patients with cStageIII disease $(P=0.010)$. This finding was not seen in the complete group or in patients with cStageI and cStageII disease (Table 3). The Ki-67-positive rate of the CR group tended to be higher than that of the Failure group in patients with cStageIII disease $(P=0.098)$; however, this did not reach statistical significance. This tendency was not seen in the overall group or in patients with cStageI or cStageII disease (Figure 2B). Representative illustrations of immunohistochemistry are presented in Figure 3.

Table 1 Characteristics of the CR group and failure group

\begin{tabular}{|c|c|c|c|c|}
\hline Variables & Total $(n=79)(\%)$ & CR group $(n=35)(\%)$ & Failure group $(n=44)(\%)$ & $P$ value* \\
\hline \multicolumn{5}{|l|}{ Age (years) } \\
\hline mean $\pm S D$ & $63.3 \pm 8.3$ & $63.6 \pm 8.8$ & $63.0 \pm 7.9$ & 0.73 \\
\hline (Range) & $(43-79)$ & $(48-79)$ & $(43-79)$ & \\
\hline \multicolumn{5}{|l|}{ Gender } \\
\hline Male & $68(86.1)$ & $30(85.7)$ & $38(86.4)$ & 1.00 \\
\hline Female & $11(13.9)$ & $5(14.3)$ & $6(13.6)$ & \\
\hline \multicolumn{5}{|l|}{ Location } \\
\hline Upper & $12(15.2)$ & $5(14.3)$ & 7 (15.9) & 0.98 \\
\hline Middle & $47(59.5)$ & $21(60.0)$ & $26(59.1)$ & \\
\hline Lower & $20(25.3)$ & $9(25.7)$ & $11(25.0)$ & \\
\hline \multicolumn{5}{|l|}{ cT } \\
\hline $\mathrm{CT1}$ & $24(30.4)$ & $19(54.3)$ & $5(11.4)$ & $<0.0001$ \\
\hline $\mathrm{cT} 2$ & $8(10.1)$ & $4(11.4)$ & $4(9.1)$ & \\
\hline cT3 & $47(59.5)$ & $12(34.3)$ & $35(22.7)$ & \\
\hline \multicolumn{5}{|l|}{$\mathrm{CN}$} \\
\hline $\mathrm{CNO}$ & $29(36.7)$ & $19(54.3)$ & $10(22.7)$ & 0.0037 \\
\hline cN1-3 & $50(63.3)$ & $16(45.7)$ & $34(77.3)$ & \\
\hline \multicolumn{5}{|l|}{ cStage } \\
\hline cStage I & $22(27.8)$ & 17 (48.6) & $5(11.4)$ & 0.0004 \\
\hline cStage II & $17(21.5)$ & $8(22.9)$ & $9(20.5)$ & \\
\hline cStage III & $40(50.6)$ & 10 (28.6) & $30(68.2)$ & \\
\hline
\end{tabular}

$\mathrm{CR}$, complete response; SD, standard deviation. 

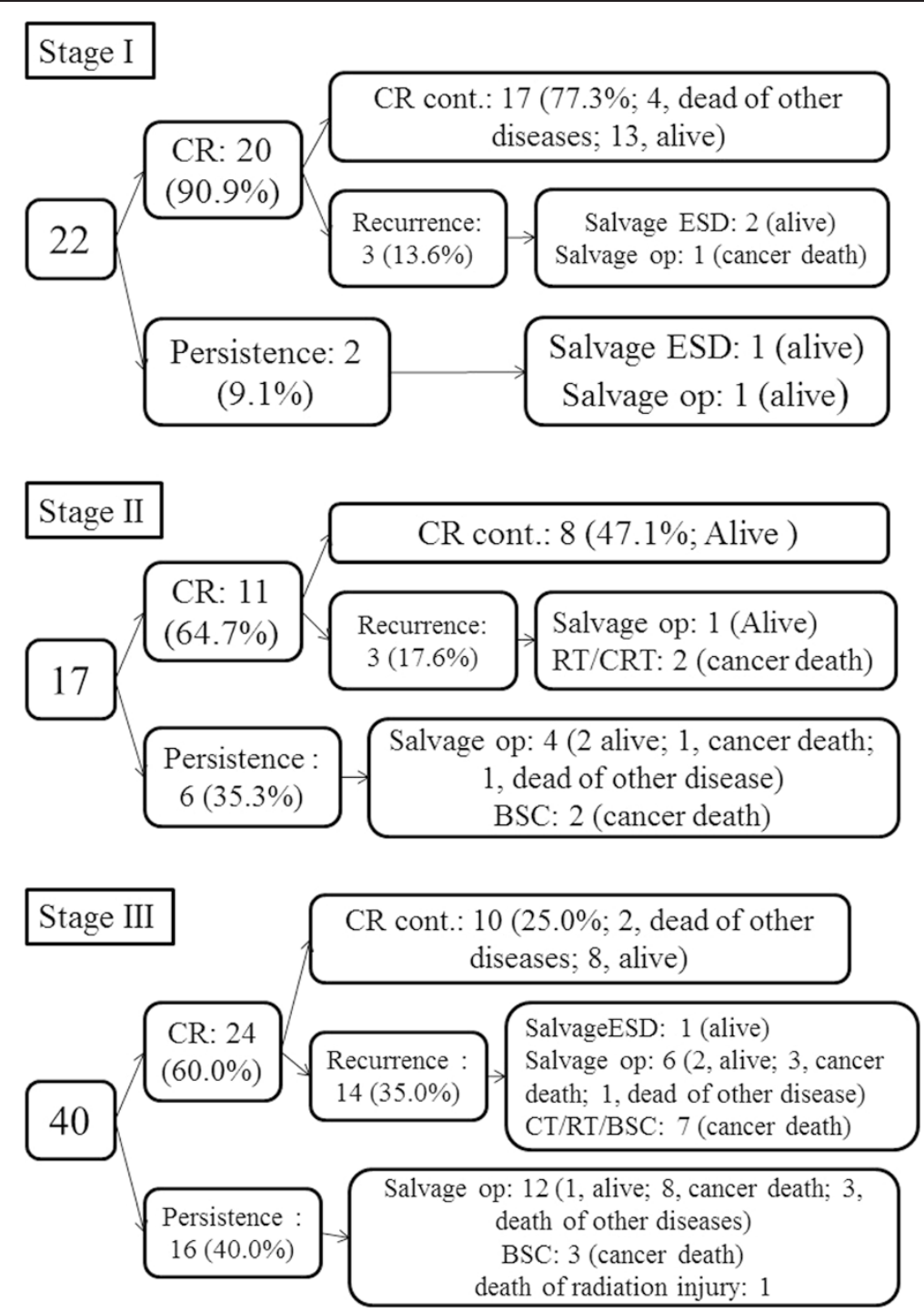

Figure 1 Clinical course of the patients according to their clinical stage. CR, complete response; CR cont., Complete response continued; ESD, endoscopic submucosal dissection; op, operation; $C T$, chemotherapy; RT, radiotherapy; $C R T$, chemoradiotherapy; BSC, best supportive care.

In terms of MDM2 expression in cStageIII, the receiver operating characteristic (ROC) curve analysis identified a cut-off for the Failure group at $9.05 \%$ that provided $90 \%$ sensitivity and $60 \%$ specificity, with a positive predictive value (PPV) of $87.1 \%$ and a negative predictive value (NPV) of $66.7 \%$. The area under the curve (AUC) was 0.76 (Figure 4A). For Ki-67 expression in cStageIII, the ROC curve analysis identified a cut-off for the CR group of $33.7 \%$ that provided $70 \%$ sensitivity and $70 \%$ specificity, with a PPV of $43.8 \%$ and a NPV of $87.5 \%$. The AUC was 0.68 (Figure 4B).

\section{Correlation between marker expressions}

In terms of correlation between marker expressions, the MDM2-positive rate tended to be higher in the p16negative group than in the p16-positive group in the overall group of patients $(P=0.021)$ and the cStageIII patients $(P=0.086)$ (Figure 5). This was not the case for cStageI and cStageII patients. No significant correlations were found among the other marker expressions with either the overall group or each cStage group (data not shown).

\section{Survival analysis to marker expression}

In terms of MDM2 positivity, the OS of the patients with MDM2 levels $\geq 9.05 \%$ was worse than that of the patients with levels $<9.05 \%$ in the overall cohort $(P=0.08)$, and patients in cStageI $(P=0.06)$, and cStageIII $(P=0.15)$; however, these numbers did not reach statistical significance (Figure 6A). The two patients with MDM2 levels $<9.05 \%$ in the cStageII group consisted of a patient who had rejected salvage treatment for persistent disease and a 
Table 2 Overall survival with clinicopathological findings

\begin{tabular}{|c|c|c|c|c|}
\hline Variables & $n=79(\%)$ & 3-years OS rate (\%) & 5 -year OS rate (\%) & $P$ value* \\
\hline \multicolumn{5}{|l|}{ Age (years) } \\
\hline$<60$ & $27(34.2)$ & 70.4 & 63.0 & 0.11 \\
\hline$\geq 60$ & $52(65.8)$ & 52.5 & 43.1 & \\
\hline \multicolumn{5}{|l|}{ Gender } \\
\hline Male & $68(86.1)$ & 58.9 & 50.8 & 0.91 \\
\hline Female & $11(13.9)$ & 57.7 & 46.2 & \\
\hline \multicolumn{5}{|l|}{ Location } \\
\hline Upper & $12(15.2)$ & 50.0 & 41.7 & control \\
\hline Middle & $47(59.5)$ & 61.0 & 48.2 & 0.76 \\
\hline Lower & $20(25.3)$ & 60.0 & 60.0 & 0.38 \\
\hline \multicolumn{5}{|l|}{ cT } \\
\hline cT1 & $24(30.4)$ & 85.9 & 76.4 & control \\
\hline cT2 & $8(10.1)$ & 85.7 & 71.4 & 0.94 \\
\hline сT3 & $47(59.5)$ & 41.5 & 34.4 & 0.0006 \\
\hline \multicolumn{5}{|l|}{$\mathrm{CN}$} \\
\hline $\mathrm{cNO}$ & $29(36.7)$ & 80.8 & 72.7 & 0.0028 \\
\hline cN1-3 & $50(63.3)$ & 47.0 & 38.2 & \\
\hline \multicolumn{5}{|l|}{ cStage } \\
\hline cStagel & $22(27.8)$ & 89.5 & 79.0 & control \\
\hline cStagell & $17(21.5)$ & 67.0 & 60.3 & 0.25 \\
\hline cStagelll & $40(50.6)$ & 40.0 & 31.8 & 0.0002 \\
\hline \multicolumn{5}{|l|}{ Clinical course } \\
\hline CR continue & $35(44.3)$ & 87.5 & 84.1 & $<0.0001$ \\
\hline Failure & $44(55.7)$ & 37.3 & 25.5 & \\
\hline
\end{tabular}

*log-rank test.

OS, overall survival.

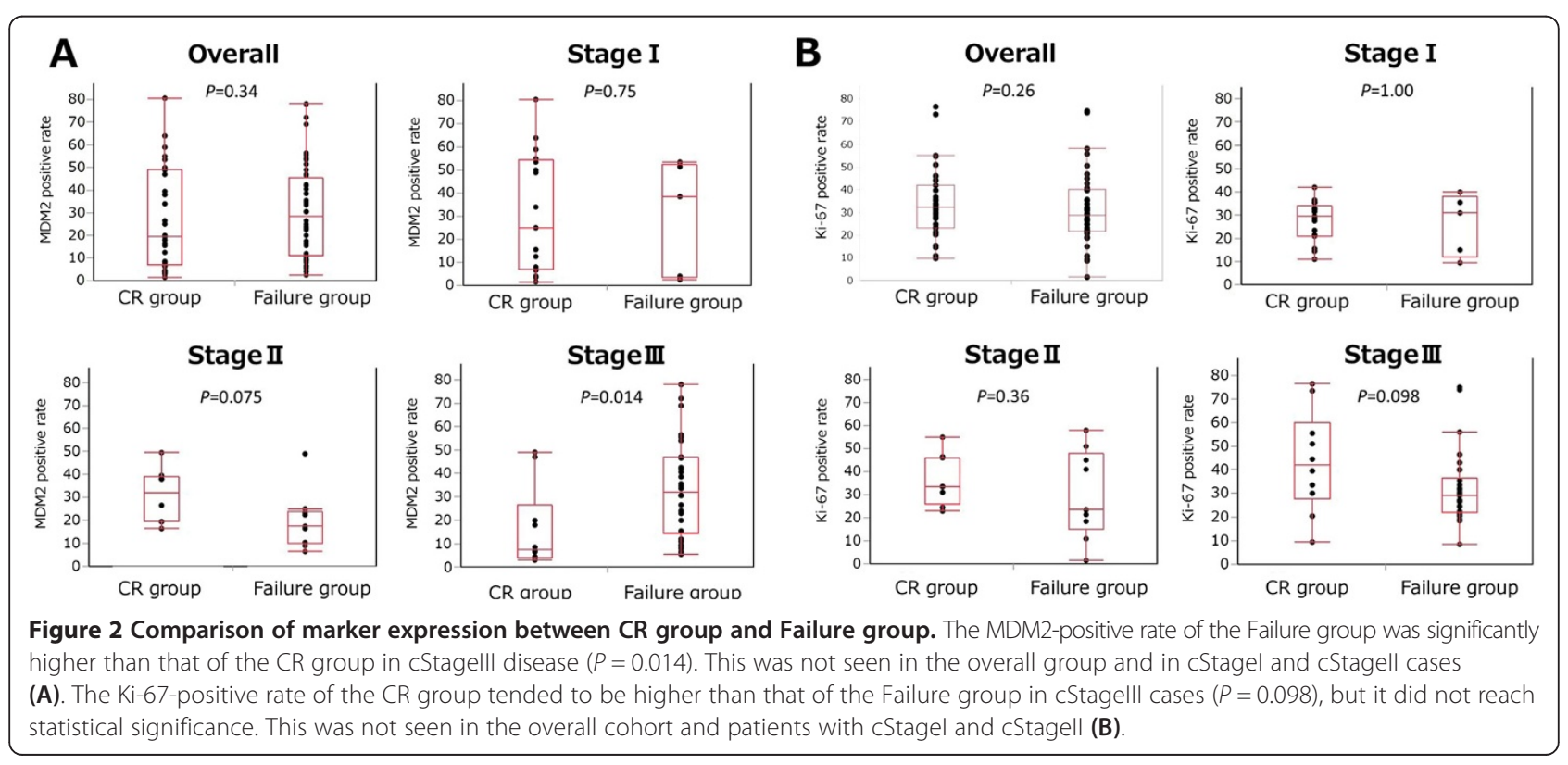


Table 3 Comparison of $p 53$ and 16 expression between the CR group and failure group

\begin{tabular}{|c|c|c|c|c|}
\hline Variables & & CR group & Failure group & $P$ value \\
\hline \multicolumn{5}{|l|}{$\overline{p 53}$} \\
\hline \multirow[t]{2}{*}{ Overall } & negative & 12 & 15 & 0.99 \\
\hline & positive & 23 & 29 & \\
\hline \multirow[t]{2}{*}{ Stagel } & negative & 6 & 0 & 0.27 \\
\hline & positive & 11 & 5 & \\
\hline \multirow[t]{2}{*}{ Stagell } & negative & 3 & 2 & 0.62 \\
\hline & positive & 5 & 7 & \\
\hline \multirow[t]{2}{*}{ Stagelll } & negative & 3 & 13 & 0.71 \\
\hline & positive & 7 & 17 & \\
\hline \multicolumn{5}{|l|}{ p16 } \\
\hline \multirow[t]{2}{*}{ Overall } & negative & 29 & 39 & 0.52 \\
\hline & positive & 6 & 5 & \\
\hline \multirow[t]{2}{*}{ Stagel } & negative & 16 & 5 & 1.00 \\
\hline & positive & 1 & 0 & \\
\hline \multirow[t]{2}{*}{ Stagell } & negative & 7 & 5 & 0.29 \\
\hline & positive & 1 & 4 & \\
\hline \multirow[t]{2}{*}{ Stagelll } & negative & 6 & 29 & 0.01 \\
\hline & positive & 4 & 1 & \\
\hline
\end{tabular}

$\mathrm{CR}$, complete response.

patient who had died of another disease after a short period. In terms of Ki-67, the OS of the patients with Ki-67 levels $\geq 33.7 \%$ was significantly better than that of patients with levels $<33.7 \%$ in the cStageIII group $(P=$ $0.024)$; however, the findings were opposite in cStageI disease $(P=0.011)$ (Figure $6 \mathrm{~B})$. The patients with Ki67 levels $\geq 33.7 \%$ in the cStageI group included a patient who underwent salvage esophagectomy for recurrent disease and died of cancer, and two patients who had continued $\mathrm{CR}$ but died of other diseases.

\section{Discussion}

The clinical course in this study showed that in earlier stages of tumor progression, the dCRT response rate was higher; moreover, the lifesaving rate of salvage treatment for the persistent and recurrent cases was also higher. In contrast, the response rate of the patients with advanced cancer, such as those with cStageIII disease, was markedly reduced and the prognosis of the patients with persistent and recurrent disease was also poorer. In addition, as the $\mathrm{cT}, \mathrm{cN}$, and cStage levels advanced, the prognosis became worse; the prognosis of the $\mathrm{CR}$ group was significantly better than that of the Failure group. These results revealed that the chemoradioresistance of ESCC may partially depend on the status of tumor progression. According to one theory, the radiosensitivity of breast cancer depends on specific factors including tumor size [14]. It would be clinically useful if we could predict the chemoradiosensitivity of ESCC using a scoring method of clinical and pathological factors such as the Van Nuys Prognostic Index in breast cancer [15]. The correlation between MDM2 expression and the prognosis of ESCC has been reportedly controversial [16-18]. However, our study suggested that MDM2 expression could be a potent predictive marker for chemoradioresistance for advanced ESCC. We may be able to consider treatment strategy while remembering that it is highly probable that cStageIII cases with high MDM2
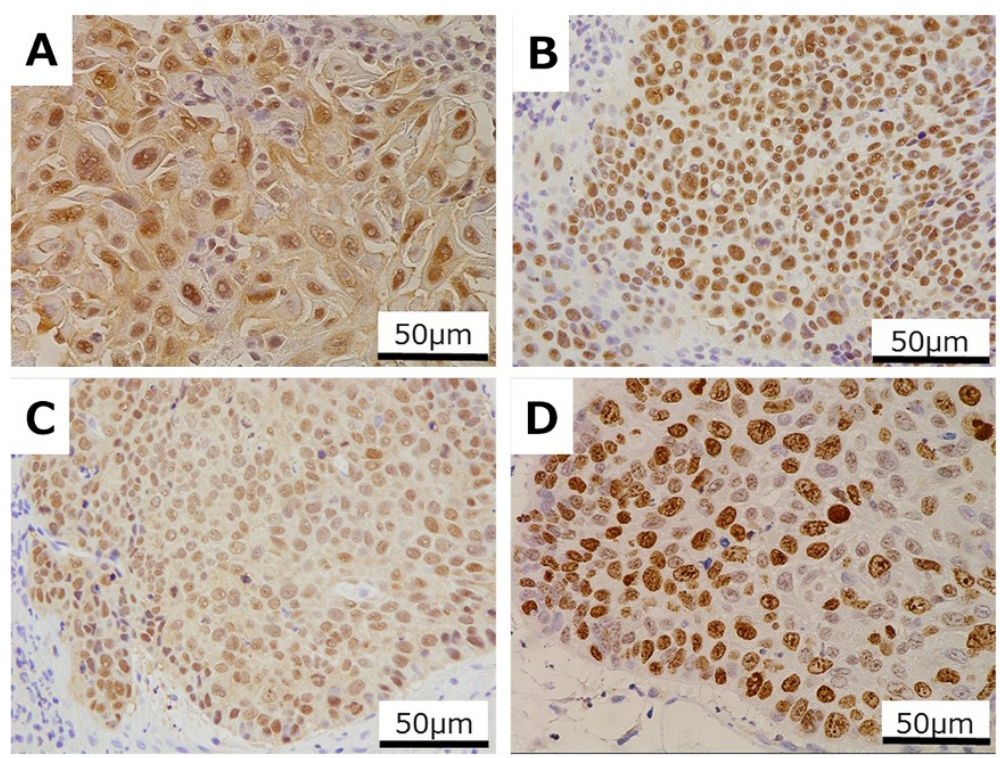

Figure 3 Immunohistochemical staining of esophageal squamous cell carcinoma. Tumor cells positive for MDM2 (A), p53 (B), p16 (C), and Ki-67 (D) expression ( $\times 400$ magnification). 

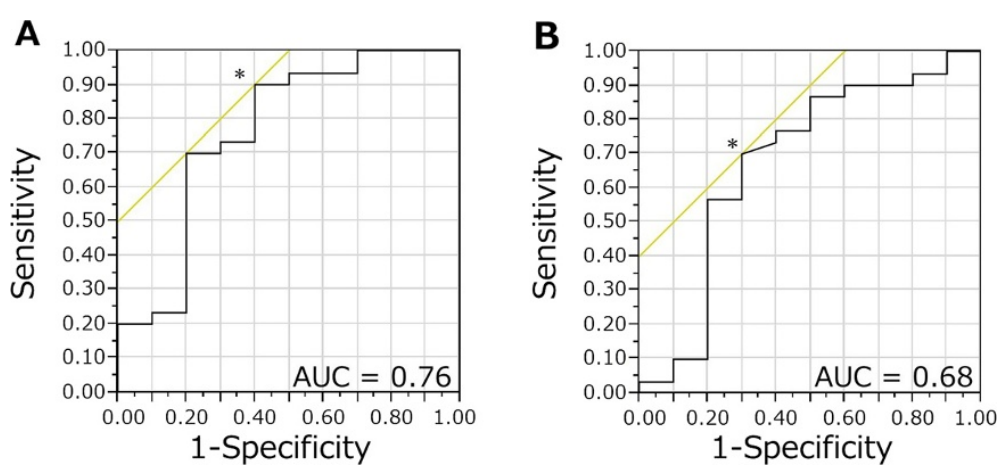

Figure 4 ROC curve of MDM2 and Ki-67 in cStagelll. The receiver operating characteristic (ROC) curve analysis of MDM2 positivity in cStagell patients identified a cut-off for the Failure group at 9.05\% that provided $90 \%$ sensitivity and $60 \%$ specificity, with a positive predictive value (PPV) of $87.1 \%$ and a negative predictive value (NPV) of $66.7 \%$. The area under the curve (AUC) was 0.76 (A). The ROC curve analysis of Ki-67 positivity in cStagelll identified a cut-off for the CR group at $33.7 \%$ that provided $70 \%$ sensitivity and $70 \%$ specificity, with a PPV of $43.8 \%$ and a NPV of $87.5 \%$. The AUC was 0.68 (B).

positivity will show chemoradioresistance. Using this strategy may suggest surgery-based treatment with/without neoadjuvant or adjuvant therapy for these cases. Although patients with high MDM2 positivity may obtain a CR state clinically, it is still necessary to closely observe them because these patients may be at high risk for cancer recurrence. The salvage treatment should be performed immediately after any clinical signs suggestive of recurrence appear, which could contribute to the improvement of the survival rate. A prospective study will be needed to evaluate these findings. Recently, MDM2 inhibitor Nutlin-3 has attracted some attention in the field of leukemia as an anti-tumor agent [19]. In addition, it has also been reported that Nutlin-3 improved the radiosensitivity of laryngeal squamous cell carcinoma, which shares many biological characteristics with ESCC [20]. It is certainly considered clinically worthwhile to attempt the clinical study of this inhibitor in patients with ESCC.

For p16, a low CRT effect was observed in p16-negative tumors only in cStageIII cases, similar to MDM2.

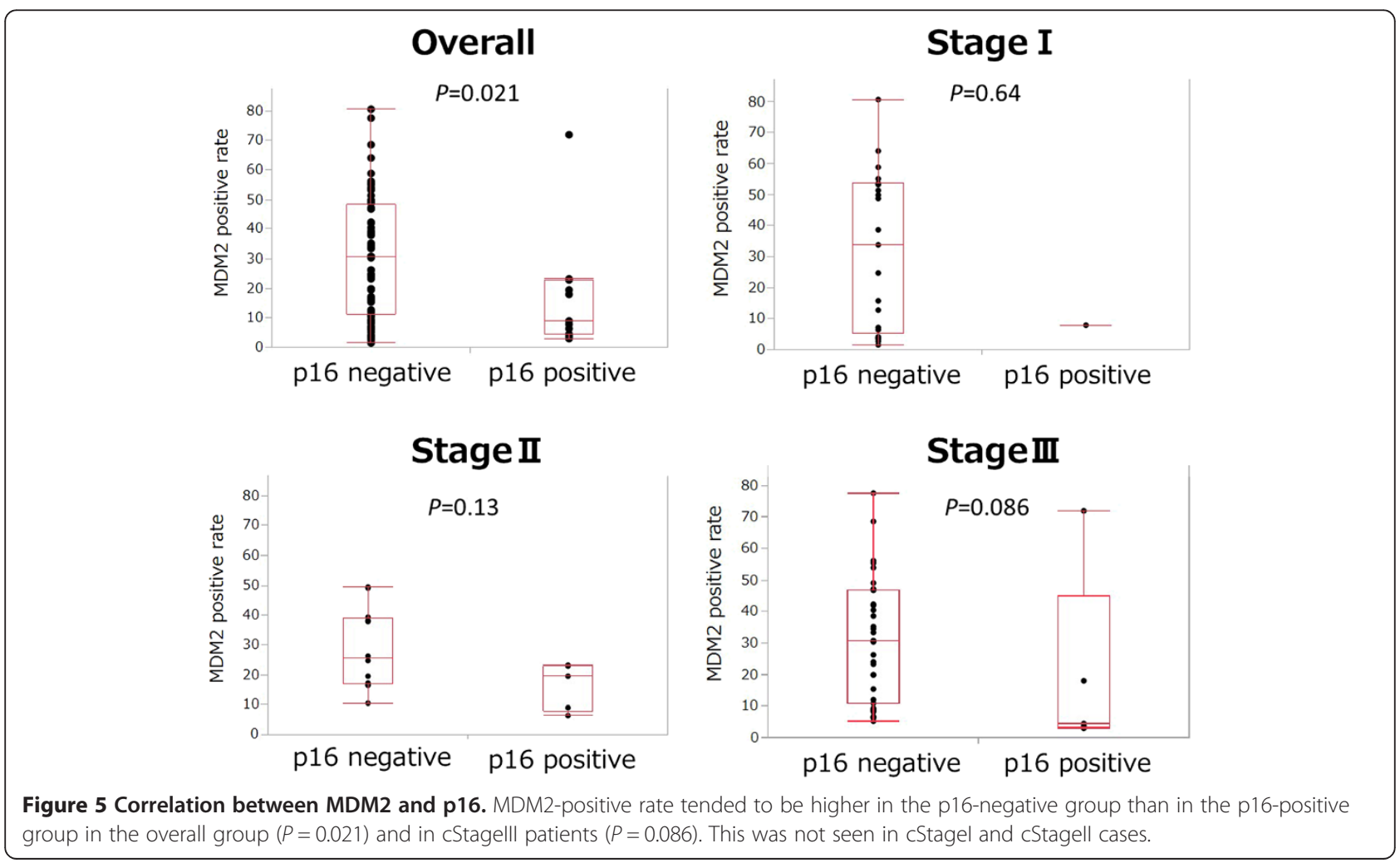




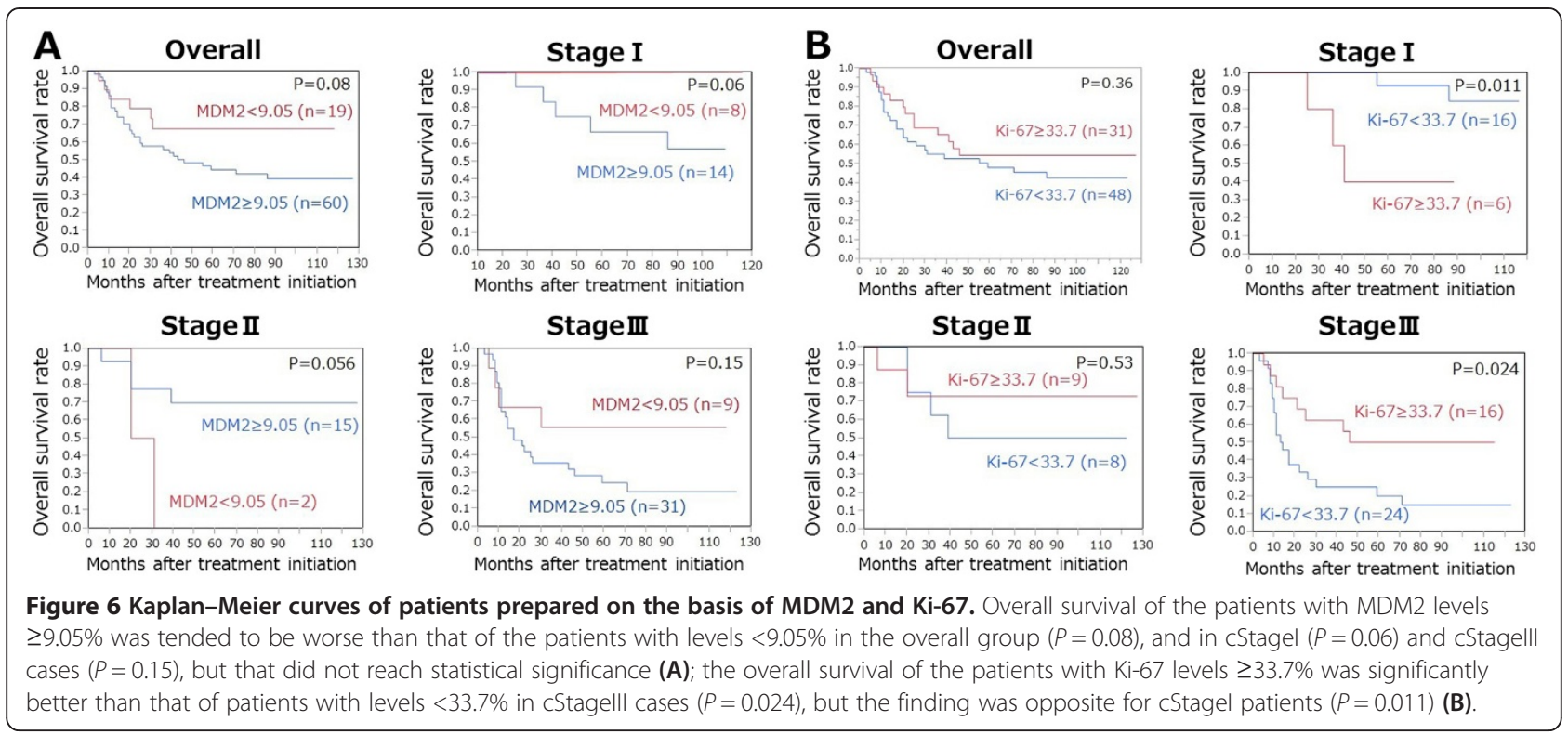

Although there were issues that will need to be addressed such as the high number of patients that were p16-negative and the small number of patients in this study, we found that p16 could also be a predictive marker for chemoradiosensitivity in advanced ESCC. No correlation was observed between chemoradiosensitivity and the status of both MDM2 and p16 in patients with cStageI and cStageII disease. Generally, the evaluation of immunostaining for dysplasia and carcinoma in situ is often difficult. MDM2 was highly expressed in so-called squamous dysplasia and carcinoma in situ [21,22]. MDM2 overexpression could play different roles in early tumorigenesis and development of chemoradioresistance or sensitivity in ESCC. Further research will be needed for the evaluation of MDM2 overexpression and its relevance to chemoradioresistance. From this point of view, the biopsy specimen should be taken from the invasive section of the tumor in advanced ESCC to completely evaluate this immunostaining.

Cut-off values between $10 \%$ and 50\% for MDM2 with respect to carcinogenesis, prognosis, and chemoradioresistance have been used [17,23-25]. We attempted to calculate the cut-off value using the ROC curve. It is conceivable that approximately $10 \%$ may be an appropriate cut-off value for prognosis and chemoradiosensitivity. Previous research has reported that high Ki-67 levels were correlated with a good response to CRT [26-28]. Imdahl et al. reported that the cut-off value of Ki-67 for responsiveness of esophageal cancer to neoadjuvant chemoradiotherapy was 39\% [27]. The cut-off value from this study (33.7\%) that was calculated using the ROC curve was also consistent with that reported by Imdahl et al. The results of the previous study for prognosis and chemoradiosensitivity followed the same trend as our current study.

Looking at the association between markers, there was no correlation between MDM2 and p53 expression in this study. The positive correlation of MDM2 and p53, and the p53-dependent and -independent role of MDM2 in ESCC has been previously reported [29,30]. Because the number of cases evaluated in this study was rather limited, further investigation will be required for these issues. On the other hand, a relationship between MDM2 and p16 has been suggested by this study. Besides inhibiting p53, MDM2 can also inhibit the cell cycle in another route; for example, MDM2 directly inhibits the retinoblastoma protein $[31,32]$. It has also been reported that both p16 and p14 (ARF), which inactivate MDM2, are on the same chromosome 9p21 [33]. However, further examination on the relationship between these peripheral markers is required.

\section{Conclusions}

In conclusion, the results of this study suggest that MDM2 and p16 might have potential as predictive markers for chemoradioresistance in cStageIII ESCC and also that Ki-67 may also have a role as a putative prognostic marker following dCRT in cStageIII ESCC. Future studies might incorporate these potential biomarkers in trials to determine their effectiveness in formulating treatment strategies for patients with advanced ESCC. We hope that this study contributes to the treatment strategy of ESCC.

\section{Abbreviations}

dCRT: Definitive chemoradiotherapy; ESCC: Esophageal squamous cell carcinoma; MDM2: Murine double minute 2; RT: Room temperature; OS: Overall survival; CR: Complete response; ROC: Receiver operating characteristic; PPV: Positive predictive value; NPV: Negative predictive value; AUC: Area under the curve.. 


\section{Competing interests}

The authors declare that they have no competing interests.

\section{Authors' contributions}

$\mathrm{HO}$ is the main author of this article. $\mathrm{HO}$ and FF conceived this study. YO, GM, TK, TN, KK, SA, YT, TS, JT, and MH made contributions to the collection of the clinical information and data analysis. $\mathrm{HO}$ performed the experiments. $\mathrm{HO}$ and FF performed the pathological examination and immunohistochemical evaluation. YN, NO, and HS reviewed the manuscript. All authors have read and approved the final manuscript.

\section{Acknowledgements}

We are grateful to the staff of the Department of Pathology, Tohoku University Hospital for their technical assistance and Enago (www.enago.jp) for the English language review.

\section{Author details}

${ }^{1}$ Department of Advanced Surgical Science and Technology, Graduate School of Medicine, Tohoku University, 1-1 Seiryo-machi, Aoba-ku, Sendai 980-8574, Japan. ²Department of Pathology, Tohoku University Hospital, Sendai, Japan. ${ }^{3}$ Department of Pathology, Graduate School of Medicine, Tohoku University, Sendai, Japan.

\section{Received: 14 September 2014 Accepted: 19 March 2015}

\section{Published online: 31 March 2015}

\section{References}

1. Kato K, Muro K, Minashi K, Ohtsu A, Ishikura S, Boku N, et al. Phase II study of chemoradiotherapy with 5-fluorouracil and cisplatin for Stage II-III esophageal squamous cell carcinoma: JCOG trial (JCOG 9906). Int J Radiat Oncol Biol Phys. 2011;81:684-90.

2. Hironaka S, Ohtsu A, Boku N, Muto M, Nagashima F, Saito H, et al. Nonrandomized comparison between definitive chemoradiotherapy and radical surgery in patients with T (2-3) N (any) M (0) squamous cell carcinoma of the esophagus. Int J Radiat Oncol Biol Phys. 2003;57:425-33.

3. Ariga H, Nemoto K, Miyazaki S, Yoshioka T, Ogawa Y, Sakayauchi T, et al. Prospective comparison of surgery alone and chemoradiotherapy with selective surgery in resectable squamous cell carcinoma of the esophagus. Int J Radiat Oncol Biol Phys. 2009;75:348-56.

4. Tachimori Y, Kanamori N, Uemura N, Hokamura N, Igaki H, Kato H. Salvage esophagectomy after high-dose chemoradiotherapy for esophageal squamous cell carcinoma. J Thorac Cardiovasc Surg. 2009;137:49-54.

5. Okamoto H, Fujishima F, Nakamura Y, Zuguchi M, Miyata G, Kamei T, et al. Murine double minute 2 and its association with chemoradioresistance of esophageal squamous cell carcinoma. Anticancer Res. 2013;33:1463-71.

6. Honda R, Tanaka H, Yasuda H. Oncoprotein MDM2 is a ubiquitin ligase E3 for tumor suppressor p53. FEBS Lett. 1997;420:25-7.

7. Momand J, Zambetti GP, Olson DC, George D, Levine AJ. The mdm-2 oncogene product forms a complex with the p53 protein and inhibits p53-mediated transactivation. Cell. 1992;69:1237-45.

8. Perry ME. Mdm2 in the response to radiation. Mol Cancer Res. 2004;2:9-19.

9. Kwong FM, Tang JC, Srivastava G, Lung ML. Inactivation mechanisms and growth suppressive effects of p16INK4a in Asian esophageal squamous carcinoma cell lines. Cancer Lett. 2004;208:207-13.

10. Serrano M, Hannon GJ, Beach D. A new regulatory motif in cell-cycle control causing specific inhibition of cyclin D/CDK4. Nature. 1993;366:704-7.

11. Wittekind C, Yamasaki S. Oesophagus including oesophagogastric junction. In: Sobin LH, Gospodarowicz MK, Wittekind C, editors. International union against cancer TNM classification of malignant tumours. 7th ed. New York: Wiley-Blackwell; 2009. p. 66-72.

12. Okumura $H$, Natsugoe $S$, Matsumoto M, Mataki $Y$, Takatori $H$, Ishigami $S$, et al. The predictive value of p53, p53R2, and p21 for the effect of chemoradiation therapy on oesophageal squamous cell carcinoma. Br J Cancer. 2005;92:284-9.

13. Taghavi N, Biramijamal F, Sotoudeh M, Khademi H, Malekzadeh R, Moaven O, et al. p16INK4a hypermethylation and p53, p16 and MDM2 protein expression in esophageal squamous cell carcinoma. BMC Cancer. 2010;10:138.

14. Silverstein MJ, Lagios MD, Groshen S, Waisman JR, Lewinsky BS, Martino S, et al. The influence of margin width on local control of ductal carcinoma in situ of the breast. N Engl J Med. 1999;340:1455-61.
15. Silverstein MJ, Lagios MD, Craig PH, Waisman JR, Lewinsky BS, Colburn WJ, et al. A prognostic index for ductal carcinoma in situ of the breast. Cancer. 1996;77:2267-74

16. Okamoto H, Fujishima F, Nakamura Y, Zuguchi M, Ozawa Y, Takahashi Y, et al. Significance of CD133 expression in esophageal squamous cell carcinoma. World J Surg Oncol. 2013;11:51.

17. Saito H, Tsujitani S, Oka S, Ikeguchi M, Maeta M, Kaibara N. The expression of murine double minute 2 is a favorable prognostic marker in esophageal squamous cell carcinoma without p53 protein accumulation. Ann Surg Oncol. 2002;9:450-6.

18. Shimada Y, Imamura M, Shibagaki I, Tanaka H, Miyahara T, Kato M, et al. Genetic alterations in patients with esophageal cancer with short- and long-term survival rates after curative esophagectomy. Ann Surg. 1997;226:162-8.

19. Drakos E, Singh RR, Rassidakis GZ, Schlette E, Li J, Claret FX, et al. Activation of the p53 pathway by the MDM2 inhibitor nutlin-3a overcomes BCL2 overexpression in a preclinical model of diffuse large B-cell lymphoma associated with t (14;18) (q32;q21). Leukemia. 2011;25:856-67.

20. Arya AK, El-Fert A, Devling T, Eccles RM, Aslam MA, Rubbi CP, et al. Nutlin-3, the small-molecule inhibitor of MDM2, promotes senescence and radiosensitises laryngeal carcinoma cells harbouring wild-type p53. Br J Cancer. 2010;103:186-95.

21. Ambrosch P, Ruschenburg I. p53 and MDM2 protein expression in squamous cell carcinoma and neighboring dysplasia from the upper aerodigestive tract. Anticancer Res. 2000;20:3151-5.

22. Stoll C, Baretton G, Lohrs U. The influence of p53 and associated factors on the outcome of patients with oral squamous cell carcinoma. Virchows Arch. 1998;433:427-33

23. Ikeguchi M, Ueda T, Fukuda K, Yamaguchi K, Tsujitani S, Kaibara N. Expression of the murine double minute gene 2 oncoprotein in esophageal squamous cell carcinoma as a novel marker for lack of response to chemoradiotreatment. Am J Clin Oncol. 2002;25:454-9.

24. Mathew R, Arora S, Khanna R, Mathur M, Shukla NK, Ralhan R. Alterations in p53 and pRb pathways and their prognostic significance in oesophageal cancer. Eur J Cancer. 2002;38:832-41.

25. Nam TK, Lee JH, Cho SH, Chung IJ, Ahn SJ, Song JY, et al. Low hMLH1 expression prior to definitive chemoradiotherapy predicts poor prognosis in esophageal squamous cell carcinoma. Cancer Lett. 2008;260:109-17.

26. Beardsmore DM, Verbeke CS, Davies CL, Guillou PJ, Clark GW. Apoptotic and proliferative indexes in esophageal cancer: predictors of response to neoadjuvant therapy apoptosis and proliferation in esophageal cancer. J Gastrointest Surg. 2003;7:77-87.

27. Imdahl A, Jenkner J, Ihling C, Ruckauer K, Farthmann EH. Is MIB-1 proliferation index a predictor for response to neoadjuvant therapy in patients with esophageal cancer? Am J Surg. 2000;179:514-20.

28. Takeuchi H, Ozawa S, Ando N, Kitagawa Y, Ueda M, Kitajima M. Cell-cycle regulators and the Ki-67 labeling index can predict the response to chemoradiotherapy and the survival of patients with locally advanced squamous cell carcinoma of the esophagus. Ann Surg Oncol. 2003;10:792-800.

29. Arora S, Mathew R, Mathur M, Chattopadhayay TK, Ralhan R. Alterations in MDM2 expression in esophageal squamous cell carcinoma: relationship with p53 status. Pathol Oncol Res. 2001;7:203-8.

30. Cheng TH, Hsu PK, Li AF, Hung IC, Huang MH, Hsu HS. Correlation of p53, MDM2 and p14 (ARF) protein expression in human esophageal squamous cell carcinoma. J Cancer Res Clin Oncol. 2009;135:1577-82.

31. Uchida C, Miwa S, Isobe T, Kitagawa K, Hattori T, Oda T, et al. Effects of MdmX on Mdm2-mediated downregulation of pRB. FEBS Lett. 2006;580:1753-8.

32. Miwa S, Uchida C, Kitagawa K, Hattori T, Oda T, Sugimura H, et al. Mdm2-mediated pRB downregulation is involved in carcinogenesis in a p53-independent manner. Biochem Biophys Res Commun. 2006;340:54-61.

33. Quelle DE, Zindy F, Ashmun RA, Sherr CJ. Alternative reading frames of the INK4a tumor suppressor gene encode two unrelated proteins capable of inducing cell cycle arrest. Cell. 1995;83:993-1000. 